\title{
Anti-mycobacterial activity of labdane and halimane diterpenes obtained from Plectranthus ornatus Codd
}

\author{
Atividade anti-micobacteriana de diterpenos de esqueleto halimano e labdano obtidos de \\ Plectranthus ornatus Codd
}

\author{
Joana M. Andrade ${ }^{a, b}$, Alessandra Romagnolic, Joana F. Almeida ${ }^{a}$, Inês M. Ferreira ${ }^{a}$, Catarina Garcia ${ }^{a, b}$, \\ Ana Díaz-Lanza ${ }^{b}$, Gian Maria Fimia ${ }^{\mathrm{c}, \mathrm{d}}$, Patrícia Rijo ${ }^{\mathrm{a}, \mathrm{e}}$ \\ aUniversidade Lusófona's Research Center for Biosciences and Health Technologies (CBIOS), Campo Grande 376, 1749- \\ 024 Lisbon, Portugal \\ ${ }^{b}$ Department of Biomedical Sciences, Faculty of Pharmacy, University of Alcalá, Campus Universitario, 28871 Alcalá de \\ Henares, Spain \\ "National Institute for the Infectious Diseases "Lazzaro Spallanzani”, Via Portuense, 292, 00149 Rome, Italy \\ ${ }^{\mathrm{d}}$ Department of Biological and Environmental Sciences and Technologies (Di.S.Te.B.A.), University of Salento, Lecce, Italy

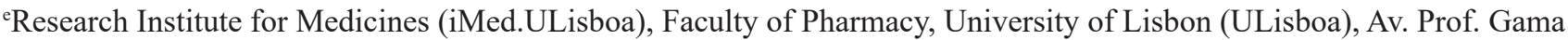 \\ Pinto, 1649-003 Lisbon, Portugal \\ Email: patricia.rijo@ulusofona.pt
}

\begin{abstract}
Natural products are a unique source of lead compounds for medicinal chemistry. Several diterpenes isolated from Plectranthus spp. have been reported to have interesting tuberculostatic activity and P. ornatus Codd. has been used in some regions of Brazil as an anti-infective.

Previously, diterpenes with halimane and labdane skeletons were isolated in large quantities from P. ornatus Codd. We assessed the anti-mycobacterial activity of these compounds, performing a preliminar assay with the non-virulent strain Mycobacterium smegmatis. The cytotoxicity of the diterpenes with halimane and labdane skeletons was tested with the lactate dehydrogenase assay, where no considerable cytotoxic effects were found up to $25 \mu \mathrm{g}$.mL-1. Subsequently, the microdilution method was used to determine the minimum inhibitory concentration (MIC) against M. smegmatis. The MIC that inhibited the growth of the non-virulent mycobacteria by $\geq 99 \%$ was $100 \mu \mathrm{g}$.mL- 1 for the diterpene with halimane skeleton, whereas for the diterpene with a labdane skeleton was $>100 \mu \mathrm{g} . \mathrm{mL}-1$.

To the best of our knowledge, this is the first report on diterpenes with halimane and labdane skeletons isolated from P. ornatus in macrophages cytotoxicity, and in a preliminar assay for anti-mycobacterial activity. Further studies are suggested on M. tuberculosis, particularly for the diterpenes with halimane skeleton.
\end{abstract}

Keywords: Plectranthus ornatus; Tuberculostatic; Mycobacterium smegmatis; Halimane; Labdane; Cytotoxicity.

\section{Resumo}

Os produtos naturais são uma fonte única de compostos-tipo para o desenvolvimento de fármacos em química medicinal. Vários diterpenos das espécies Plectranthus foram referidos com actividade tuberculostática interessante, sendo que o P. ornatus Codd. é usado como anti-infeccioso em algumas regiões do Brasil.

Em trabalhos anteriores, um diterpeno de esqueleto de halimano outro labdano foram isolados a partir de P. ornatus Codd. Neste trabalho, avaliou-se preliminarmente a sua actividade micobacteriana com uma estirpe não virulenta de Mycobacterium smegmatis.

A citotoxicidade dos compostos foi testada medindo a libertação de lactato desidrogenase (LDH) não sendo encontrados efeitos citotóxicos consideráveis até $25 \mu \mathrm{g} / \mathrm{mL}$. Posteriormente, o método de microdiluição foi utilizado para determinar a concentração mínima inibitória (CMI) de M. smegmatis. A CMI $\geq 99 \%$ para o esqueleto de halimano foi $100 \mu \mathrm{g} / \mathrm{mL}$ e $>100 \mu \mathrm{g} / \mathrm{mL}$ para o esqueleto de labdano.

De acordo com o nosso conhecimento, este é o primeiro estudo usando diterpenos de esqueleto de halimano e de labdano isolados a partir de P. ornatus em ensaios de citotoxicidade em macrófagos, e num ensaio preliminar sobre a sua atividade anti-micobacteriana. Estudos futuros são sugeridos na estirpe virulenta de M. tuberculosis, particularmente para os diterpenos de esqueleto de halimano.

Palavras chave: Plectranthus ornatus; Tuberculostático; Mycobacterium smegmatis; Halimano; Labdano; Citotoxicidade. 


\section{Introduction}

Tuberculosis (TB) is one of the major causes of death from infectious disease worldwide, according to the World Health Organization (WHO). The causative agent, Mycobacterium tuberculosis (Mtb) most often affects the lungs, and has developed elaborate survival mechanisms in the human host, allowing it to remain in a clinically latent infection state before eventually progressing to the active disease (1-4). TB is still considered as a public health problem for many reasons. One third of the world's population is infected with the mycobacteria, and the means for diagnosis and treatment in African and Asian countries are very poor. Additionally, there have been reports of multidrug-resistant (MDR) TB strains that do not respond to isoniazid and rifampicin treatment 5,6). The WHO estimates 600000 new cases of TB resistant to rifampicin - the most effective first-line drug - and approximately $6.2 \%$ of MDR-TB cases were considered to be extensively drug-resistant (XDR)-TB (7).

Under these circumstances, the identification of new therapeutic agents remains an essential achievement in order to control Mtb infection and reduce deaths resulting from TB. I Recently, there has been a renewed interest in compounds of natural origin to find TB lead compounds. Natural products are a unique source of compounds for medicinal chemistry drug development, as they present complex chemical structures which may be important for specific interactions and recognition of potential macromolecular targets in the pathogenic microorganisms (8).

M. smegmatis is an easy to grow, non-pathogenic mycobacterium often used as a first approach to anti-tubercular studies, as it is an attractive model organism of the pathogen M. tuberculosis (9). Although it is not possible to reach a conclusion regarding efficacy for treatment of tuberculosis without testing on the $M$. tuberculosis strain (10), M. smegmatis is particularly useful in understanding the cellular processes that are important to pathogenic mycobacteria (11).

The Plectranthus genus belongs to the Lamiaceae family and is widely distributed in tropical Africa, Asia and Australia. This genus comprises many plants of medicinal, ornamental and economic interest, with a rich diversity of ethnobotanical uses in several ailments such as skin, respiratory and gastrointestinal complaints, pain, infections and fever (12-14). The isolation of the bioactive metabolites from Plectranthus spp. is important to validate the popular use of these plants. The constituents of Plectranthus plants are primarily halimane, abietane, kaurane, clerodane and labdane diterpenoids,

\section{Introdução}

A tuberculose (TB) é uma das principais causas de morte por doença infecciosa em todo o mundo, de acordo com a Organização Mundial da Saúde (OMS). $\mathrm{O}$ agente causador, Mycobacterium tuberculosis (Mtb), afeta os pulmões mais frequentemente e desenvolveu mecanismos de sobrevivência elaborados no hospedeiro humano, permitindo que ele permaneça num estado de infecção clinicamente latente, antes de eventualmente progredir para a doença ativa (1-4). A tuberculose ainda é considerada um problema de saúde pública por muitas razões, a primeira porque um terço da população mundial está infectada com micobactérias e os meios para diagnóstico e tratamento em países africanos e asiáticos são muito pobres; em segundo lugar, houve relatos de TB resistente a múltiplos medicamentos (MDR) que não respondem ao tratamento nem da isoniazida nem da rifampicina $(5,6)$. A OMS estima que há 600000 novos casos com resistência à rifampicina - o medicamento de primeira linha mais eficaz e cerca de $6,2 \%$ dos casos de TB-MDR apresentaram extensa resistência a medicamentos (XDR) em 2016 (7).

Nestas circunstâncias, a identificação de novos agentes terapêuticos continua a ser uma conquista essencial para controlar a infecção por Mtb e reduzir as mortes por tuberculose. Na verdade, houve um interesse renovado em compostos de origem natural para encontrar compostos protótipo (para o tratamento da tuberculose) para o desenvolvimento de fármacos em química medicinal. Estes compostos apresentam estruturas químicas complexas que podem ser importantes para interações específicas e reconhecimento de potenciais alvos macromoleculares nos microrganismos patogénicos (8).

M. smegmatis é uma micobactéria não patogênica, fácil de semear, e frequentemente usada como primeira abordagem para estudos anti-tuberculares, pois é um bom organismo modelo do patogéneo mundial $M$. tuberculosis (9). Embora não seja possível chegar a uma conclusão sobre o tratamento da tuberculose sem testar a estirpe de M. tuberculosis (10). O M. smegmatis é particularmente útil na compreensão dos processos celulares que são importantes para micobactérias patogénicas (11).

Os plantas do género Plectranthus pertencem à família Lamiaceae e estão amplamente distribuídas na África tropical, Ásia e Austrália. Estas plantas são de grande interesse medicinal e usos etnofarmacológicos, incluindo doenças cutâneas, respiratórias e gastrointestinais, dor, infecções e febre (12-14). É importante validar o uso popular destas plantas através do isolamento dos 
together with ursane, oleanane and lupane triterpenoids, phytosterols, flavonoids and other polyphenolic compounds $(12,15,16)$. There are many Plectranthus spp. with characteristic antimicrobial activity in both extracts and compounds. This work will be focused on $P$. ornatus, a medicinal plant used for its antibiotic action (17).

In previous works, the halimane diterpene obtained from the $P$. ornatus acetone extract has been reported to have antimicrobial activity against Gram-positive bacteria, such as M. smegmatis (17).

Given the promising ethnopharmacology of P. ornatus, this work aimed to perform a preliminary anti-tubercular evaluation of one halimane and two labdane compounds previously isolated from this medicinal plant. The anti-tubercular activity was assessed through the determination of the minimum inhibitory concentration (MIC) that inhibited the growth of the mycobacteria by $\geq 99 \%\left(\mathrm{MIC}_{99}\right)$, after cytotoxic evaluation on macrophage cells isolated from PBMC.

\section{Materials and methods}

\section{Reagents and materials}

Rifampicin, Triton X-100, Tween 80 and phosphate buffered saline (PBS) were purchased from SigmaAldrich. Phosphoric acid and dimethyl sulphoxide (DMSO) were purchased from Merck. Anti-CD14 conjugated magnetic microbeads were provided by Miltenyi. Lymphoprep was obtained from Cedarlane. CytoTox $^{\circledR} 96$ non-radioactive cytotoxicity assay kit (G1780) was from Promega.

\section{Plant material}

The medicinal plant $P$. ornatus Codd. originated from South Africa, and was cultivated in Instituto Superior de Agronomia campus (Lisbon). The plant voucher specimen was identified by Eurico S. Martins, and was deposited in the Herbarium of the Instituto de Investigação Cientifica Tropical, Lisbon (ref. C. Marques S/ $\mathrm{N}^{\mathrm{o}}$ LISC).

\section{Microbial strain}

M. smegmatis (ATCC 607) was grown at $37^{\circ} \mathrm{C}$, with shaking, in Middlebrook 7H9 broth (Difco) supple- metabolitos bioativos de Plectranthus spp. Os constituintes das plantas de Plectranthus são principalmente diterpenos de halimano, abietano, kaurano, clerodano e labdano, juntamente com triterpenóides de ursano, oleanano e triterpenóides de lupano, fitosteróis, flavonóides e outros compostos polifenólicos $(12,15,16)$. Existem muitas espécies de Plectranthus com atividade antimicrobiana característica em extratos e compostos. Assim, este trabalho será focado em $P$. ornatus, uma planta medicinal usada para doenças digestivas e cujas as folhas são usadas devido à sua ação antibiótica (17). Trabalhos anteriores relataram a atividade antimicrobiana de um composto de $P$. ornatus, o diterpeno de halimano obtido a partir do extrato de acetona, e que já foi relatado com atividade antimicrobiana contra bactérias de Gram-positivo, como o M. smegmatis (17).

Dada a etnofarmacologia promissora de $P$. ornatus, este trabalho teve como objetivo a realização de uma avaliação preliminar anti-tubercular de um halimano e de dois compostos de labdano, previamente isolados desta planta medicinal. A atividade anti-tubercular foi avaliada através da determinação da concentração mínima inibitória (CMI), que inibiu o crescimento das micobactérias em $\geq 99 \%\left(\mathrm{CMI}_{99}\right)$, após avaliação citotóxica em células de macrófagos isoladas de PBMC.

\section{Materiais e métodos}

\section{Reagentes e materiais}

Rifampicina, Triton X-100, Tween 80 e solução tamponada de fosfatos (PBS) foram adquiridos a Sigma-Aldrich. O ácido fosfórico e o dimetil sulfóxido (DMSO) foram adquiridos da Merck. As microbeads magnéticas conjugadas anti-CD14 foram fornecidas por Miltenyi. O Lymphoprep foi obtido em Cedarlane. O kit de análise de citotoxicidade não-radioativa CytoTox ${ }^{\circledR} 96$ (G1780) foi proveniente da Promega.

\section{Material vegetal}

A planta medicinal Plectranthus ornatus Codd., era original da África do Sul, e foi cultivado no campus do Instituto Superior de Agronomia (Lisboa). O voucher do espécime da planta foi identificado por Eurico S. Martins, e depositados no Herbário do Instituto de Investigação Cientifica Tropical, em Lisboa (ref. C. Marques $\mathrm{S} / \mathrm{N}^{\mathrm{o}}$ LISC).

\section{Estirpe microbiana}

O Mycobacterium smegmatis (ATCC 607) foi cultivado a $37^{\circ} \mathrm{C}$, com agitação, em meio Middlebrook $7 \mathrm{H} 9$ 
mented with $10 \%$ albumin-dextrose-catalase (ADC), $0.2 \%$ glycerol, and $0.05 \%$ Tween 80 .

\section{Compound isolation}

The halimane compound $\left(11 R^{*}, 13 E\right)$-11-acetoxyhalima-5,13-dien-15-oic acid [Compound 1], the labdane diterpenoids Plectrornatine $\mathrm{C}$ [Compound 2] and the forskolin-like 1:1 mixture of 1,6-di- $O$-acetylforskolin and 1,6-di-O-acetyl-9-deoxyforskolin [Compound 3], were previously isolated from Plectranthus ornatus Codd. (18).

The compounds were obtained by a phytochemical study that was previously performed in the described plant, using bioguided studies (18-21). The structural elucidation of the bioactive metabolites was based on physicochemical data (melting point, specific rotation), spectroscopic data (UV, IR, 1D- and 2D- ${ }^{1} \mathrm{H}$ and ${ }^{13} \mathrm{C}$ NMR), mass spectra, elemental analysis, and comparison with bibliographic data.
(Difco) suplementado com 10\% de albumina-dextrosecatalase (ADC), 0,2\% de glicerol e $0,05 \%$ de Tween 80 .

\section{Isolamento de compostos}

O ácido (11 $\left.R^{*}, 13 E\right)$-11-acetoxihalima-5,13-dien-15-óico (1), os diterpenos de labdano Plectrornatina C (2) e os compostos semelhantes a forscolinas, mistura 1:1 de 1,6-di- $O$-acetilforscolina e 1,6-di- $O$-acetil-9-desoxiforscolina (3), foram previamente isolados de Plectranthus ornatus Codd. (18).

Os compostos foram obtidos por um estudo fitoquímico que foi previamente realizado nesta planta, utilizando estudos bioguiados (18-21). A elucidação estrutural dos metabolitos bioativos baseou-se em dados físicoquímicos (ponto de fusão, rotação específica), principalmente espectroscópicos UV, IR, 1D e espectros 2D${ }^{1} \mathrm{H} \mathrm{e}{ }^{13} \mathrm{C}-\mathrm{RMN}$, espectros de massa, análise elementar e comparação com dados bibliográficos.

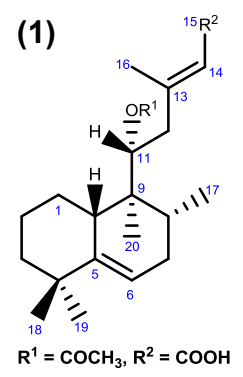

(2)

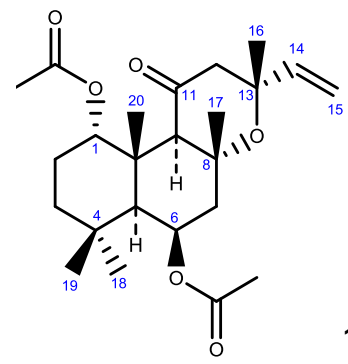

(3)

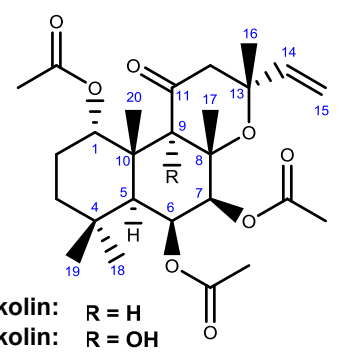

Figure 1 - Halimane (1) and labdane (2-3) diterpenes isolated from Plectranthus ornatus, under study.

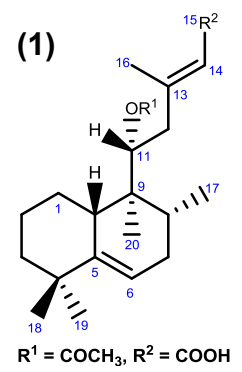

(2)

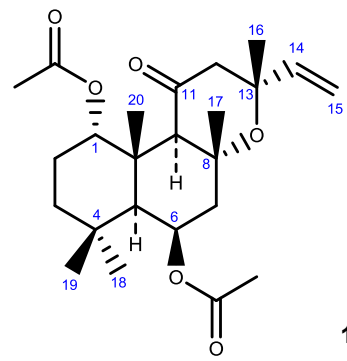

(3)

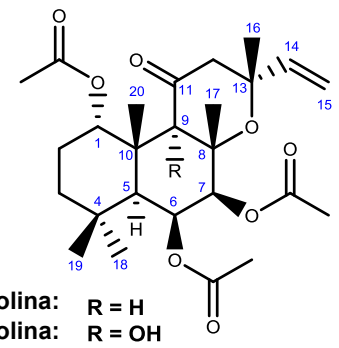

Figura 1 - Diterpenos de esqueleto de halimano (1) e labdano (2-3) em estudo, isolados de Plectranthus ornatus, em estudo. 


\section{Cytotoxic evaluation}

\section{Peripheral blood mononuclear cell isolation}

The peripheral blood mononuclear cells (PBMC) isolation was performed as previously described $(22,23)$. Briefly, PBMC were isolated from freshly collected buffy coats obtained from healthy voluntary blood donors by Ficoll density gradient centrifugation using Lymphoprep. Monocytes were purified by positive sorting using anti-CD14 conjugated magnetic microbeads. The recovered cells were $>99 \% \mathrm{CD}_{14}{ }^{+}$as determined by flow cytometry with anti-CD14 antibody. Monocytes were cultured in 48 -well plates at $5.0 \times 10^{5}$ cells $/ \mathrm{mL}$ in X-VIVO medium supplemented with $2 \%$ human serum and incubated for five days at $37{ }^{\circ} \mathrm{C}$ in a $5 \% \mathrm{CO}_{2}$ humidified atmosphere. No antibiotics were added to the cultures.

\section{LDH assay}

The cytotoxicity of Compounds $\mathbf{1}$ and $\mathbf{2}$ to exposed human macrophages was evaluated by the lactate dehydrogenase (LDH) assay using the CytoTox $96^{\circledR}$ NonRadioactive Cytotoxicity Assay Kit (Promega G1780). After 48 hours of cell adhesion, the cells were treated with the compounds at concentrations corresponding to approximate MIC values, tested in previous works against $M$. smegmatis $(25 \mu \mathrm{g} / \mathrm{mL})(17,24)$. In addition to the kit lysis solution consisting of $9 \%(\mathrm{v} / \mathrm{v})$ Triton $\mathrm{X}-100$ in water, corresponding to $100 \% \mathrm{LDH}$ release, staurosporine $(2 \mu \mathrm{M})$ was used as positive control. The supernatants were prepared in two dilutions $(1: 10$ and 1:100). Release of LDH was measured by supplying lactate, $\mathrm{NAD}^{+}$and iodonitrotetrazolium (INT) violet as substrates in the presence of diaphorase. The substrate mixture was prepared using the assay buffer (PBS containing $0.2 \mathrm{~g} / \mathrm{L} \mathrm{KCl}, 8.0 \mathrm{~g} / \mathrm{L} \mathrm{NaCl}, 0.2 \mathrm{~g} / \mathrm{L} \mathrm{KH}_{2} \mathrm{PO}_{4}$, $1.15 \mathrm{~g} / \mathrm{L} \mathrm{Na}_{2} \mathrm{HPO}_{4}$, and $1 \%$ (w/v) bovine serum albumin) according to the manufacturer's instructions and $50 \mu \mathrm{L}$ were added to each well and incubated for 30 minutes in the dark. After incubation, $50 \mu \mathrm{L}$ of stop solution (1 $\mathrm{M}$ acetic acid) were added and quantification of the red formazan product was performed from absorbance measurements at $490 \mathrm{~nm}$ in a microplate reader. A maximum $\mathrm{LDH}$ release control was also performed by freezing the plates at $-80{ }^{\circ} \mathrm{C}$ followed by heating at $50{ }^{\circ} \mathrm{C}$.

\section{Avaliação citotóxica}

Isolamento de células mononucleares de sangue periférico (PBMC)

$\mathrm{O}$ isolamento das células mononucleares do sangue periférico $(\mathrm{PBMC})$ foi realizado como descrito anteriormente $(22,23)$. Resumidamente, as PBMC foram isoladas de doadores voluntários de sangue (saudáveis), e obtidas por centrifugação com gradiente de densidade de Ficoll usando Lymphoprep. Os monócitos foram purificados por triagem positiva utilizando microbeads anti-CD14 magnéticas conjugadas. As células recuperadas foram $>99 \% \mathrm{CD}^{+} 4^{+}$, como determinado por citometria de fluxo com anticorpo anti-CD14. Os monócitos foram cultivados em placas de 48 poços a 5,0 $0 \times 10^{5}$ células/mL em meio X-VIVO suplementado com $2 \%$ de soro humano, e incubadas durante 5 dias a $37{ }^{\circ} \mathrm{C}$ numa atmosfera de $\mathrm{CO}_{2}$ humidificada a $5 \%$. Nenhum antibiótico foi adicionado às culturas.

\section{Ensaio $\mathrm{LDH}$}

A citotoxicidade dos compostos $\mathbf{1}$ - $\mathbf{2}$ expostos aos macrófagos humanos foi avaliada pelo ensaio do lactato desidrogenase (LDH), usando o kit de ensaio de citotoxicidade não radioativa CytoTox 96 (Promega G1780). Após $48 \mathrm{~h}$ de adesão celular, as células foram incubadas com os compostos em concentrações aproximadas da concentração miníma inibitória (CMI), testados em trabalhos anteriores contra M. smegmatis $(25 \mu \mathrm{g} / \mathrm{mL})$ $(17,24)$. Além da solução de lise do kit constituída por $9 \%(\mathrm{v} / \mathrm{v})$ de Triton X-100 em água, correspondente à libertação de $100 \%$ de LDH, a estaurosporina $(2 \mu \mathrm{M})$ foi também usada como controlo positivo. Os sobrenadantes foram preparados em 2 diluições (1:10 e 1: 100). A libertação de LDH foi medida fornecendo lactato, $\mathrm{NAD}^{+}$e iodonitrotetrazólio (INT) violeta como substratos na presença de diaforase. A mistura de substrato foi preparada utilizando o tampão de ensaio (PBS contendo 0,2 g/L KCl, 8,0 g/L NaCl, 0,2 g/L KH $\mathrm{PO}_{4}$, $1,15 \mathrm{~g} / \mathrm{L} \mathrm{Na}_{2} \mathrm{HPO}_{4}$ e $1 \%(\mathrm{~m} / \mathrm{v})$ de albumina de soro bovino) de acordo com instruções de fabricante, e foram adicionados $50 \mu \mathrm{L}$ a cada poço e incubados durante 30 minutos no escuro. Após a incubação, adicionou-se 50 $\mu \mathrm{L}$ de solução stop (ácido acético $1 \mathrm{M}$ ) e a formação do produto formazano vermelho foi quantificada a partir de medições de absorvência a $490 \mathrm{~nm}$, num leitor de microplacas. Foi também realizado um controlo de libertação máxima de $\mathrm{LDH}$, congelando as placas a -80 ${ }^{\circ} \mathrm{C}$ e reaquecendo-as a $50{ }^{\circ} \mathrm{C}$. 
Microdilution method for anti-mycobacterial evaluation

The minimum inhibitory concentrations (MICs) that inhibited the growth of the mycobacteria by $\geq 99 \%\left(\mathrm{MIC}_{99}\right)$ was determined by means of the twofold serial broth microdilution assay (25). The compounds, dissolved in DMSO, were diluted with Mueller-Hinton broth medium at concentrations ranging from 100 to $0.097 \mu \mathrm{g} /$ $\mathrm{mL}$. The antimicrobial activity of the solvent DMSO was evaluated, and rifampicin was used as control. The MIC values were taken as the lowest concentration of the compound that inhibited $\geq 99 \%$ the growth of the microorganisms, after 24 hours of incubation at $37{ }^{\circ} \mathrm{C}$, and are presented in $\mu \mathrm{g} / \mathrm{mL}$. The microbial growth was measured with an Absorbance Microplate Reader set to $620 \mathrm{~nm}$ (Thermo Scientific Multiskan FC). Assays were carried out in triplicate.
Método de microdiluição para avaliação antimicobacteriana

As concentrações inibitórias mínimas (CMI) que inibiram o crescimento das micobactérias em $\geq 99 \%\left(\mathrm{CMI}_{99}\right)$ foram determinadas por meio do ensaio de diluição seriada 1:2 (25). Os compostos, dissolvidos em DMSO, foram diluídos em concentrações variando de 100 a 0,097 $\mu \mathrm{g} / \mathrm{mL}$, com meio de Mueller-Hinton. Foi avaliada a atividade antimicrobiana do solvente DMSO. A Rifampicina foi utilizada como controlo. Os valores de CMI foram tomados como a menor concentração do composto que inibiu $\geq 99 \%$ o crescimento dos microrganismos, após $24 \mathrm{~h}$ de incubação a $37^{\circ} \mathrm{C}$, e estão apresentados em $\mu \mathrm{g} / \mathrm{mL}$. O crescimento microbiano foi medido com um leitor de microplacas de absorção ajustado para $620 \mathrm{~nm}$ (Termo Scientific Multiskan FC). Os ensaios foram realizados em triplicado.

Table 1/ Tabela 1 - LDH cytotoxicity assay for halimane (1) and labdane (2) diterpenes isolated compounds from P. ornatus, tested at their MIC values. Negative control (untreated cells); Positive control $9 \%(\mathrm{v} / \mathrm{v})$ Triton X-100 (100 \% of LDH release), STS, staurosporine $(2 \mu \mathrm{M})$, positive control. Data expressed as median $\pm \mathrm{SD}(\mathrm{n}=3) ; * * \mathrm{p}<0.0001$. For compound number chemical assignment, refer to Figure 1/ Ensaio de citotoxicidade LDH para os diterpenos halimano (1) e labdanos (2) isolados de $P$. ornatus, testados com os valores de MIC. Controlo negativo (células não tratadas); Controlo positivo $9 \%$ (v / v) Triton X-100 (100\% da libertação de LDH), STS, staurosporina (2 $\mu \mathrm{M})$, controlo positivo. Dados expressos como mediana $\pm \mathrm{SD}(\mathrm{n}=3) ; * * * \mathrm{p}<0,0001$. Para a atribuição química do número composto, consultar a Figura 1.

\begin{tabular}{c|c}
$\begin{array}{c}\text { Compound/Composto } \\
\text { STS }\end{array}$ & $\begin{array}{c}\text { LDH Cytotoxicity/ Citotoxicidade } \\
\mathbf{( \% )}\end{array}$ \\
\hline $\begin{array}{c}\text { Positive control (kit)/Controlo } \\
\text { Positivo }\end{array}$ & $21.9^{* * *}$ \\
Negative control/control negativo & 82.9 \\
$\mathbf{1}$ & 10.1 \\
$\mathbf{2}$ & 8.75 \\
$\mathbf{3}$ & 9.82 \\
\end{tabular}




\section{Statistical analysis}

Data comparison was conducted with one-way analysis of variance (ANOVA) followed by post-hoc Tukey honest significant difference test, for pairwise comparisons, using GraphPad Prism software version 5 (GraphPad Software, Inc., San Diego, California, USA). Values of $p<0.05$ were considered statistically significant. All assays were performed at least in triplicate and results are presented as median \pm standard deviation (SD).

\section{Results and Discussion}

\section{Anti-mycobacterial activity}

Quantitative analysis of $M$. smegmatis growth via the determination of MIC values was the method of choice to evaluate the anti-mycobacterial activity of the selected diterpene compounds, Compounds 1-3). Although it is not the causative agent of tuberculosis, $M$. smegmatis stands as a good preliminary model for the evaluation of possible new therapeutics compounds, to be further studied against tuberculosis $(9,10)$.

In order to avoid false positives resulting from cytotoxicity of the compounds to the cells, a cytotoxic evaluation was performed. The cytotoxicity assay measured the release of cytosolic enzyme LDH after treatment of macrophages, derived from PBMC, with Compounds 1-2. Staurosporine $\left(\mathrm{IC}_{50}=250 \mathrm{nM}\right)$, a known protein kinase inhibitor that induces apoptosis, was used as the cytotoxicity positive control (26). The results obtained are shown in Table 2.

As shown in Table 2, the staurosporine positive control resulted in macrophage cytotoxicity of $21.86 \%$ ( $p<$ $0.0001)$. In contrast, the compounds tested did not reveal significant toxicity toward macrophage cells $(p>$ $0.05)$, since cell viability was similar to values obtained for untreated cells used as negative control.

Compounds 1-3 did not show activity against $M$. smegmatis (Table 2) (MIC values of $\geq 100 \mu \mathrm{g} / \mathrm{mL}$ ), in comparison with the positive control Rifampicin (MIC $3.75 \mathrm{ng} / \mathrm{mL}$ ). Although, in comparison with the labdane diterpenes [Compound 2] and [Compound 3], the halimane diterpene [Compound 1] was the most active with a MIC of $100 \mu \mathrm{g} / \mathrm{mL}$, suggesting that further studies, such as prepararation of a pharmaceutical formulation of new derivatives from the pure halimane and testing on $M$. tuberculosis, should be made to understand its anti-tubercular activity and improve its efficacy.
Análise estatística

A comparação de dados foi realizada com uma análise de variância unidirecional (ANOVA), seguida do teste pos-hoc de diferença significativa honesta Tukey, para comparações em pares, usando o software GraphPad Prism versão 5 (GraphPad Software, Inc., San Diego, Califórnia, EUA). Os valores de $p<0,05$ foram considerados estatisticamente significativos. Todos os ensaios foram realizados pelo menos em triplicado e os resultados são apresentados como mediana \pm desvio padrão (SD).

\section{Resultados e discussão}

\section{Atividade anti-micobacteriana}

O método de escolha para avaliar a atividade anti-micobacteriana dos compostos diterpenos selecionados 1-3 foi feita através da análise quantitativa do crescimento de M. smegmatis através da determinação dos valores de CMI. Embora este não seja o agente causador da tuberculose, M. smegmatis é um bom modelo preliminar para a avaliação de possíveis novos agentes, a serem avaliados contra a tuberculose $(9,10)$.

Para evitar falsos positivos resultantes da citotoxicidade dos compostos nas células, realizou-se uma avaliação citotóxica. $\mathrm{O}$ teste de citotoxicidade mediu a libertação da enzima citosólica LDH após o tratamento dos macrófagos, derivados de PBMC, com os compostos 1-2. A estaurosporina $\left(\mathrm{IC}_{50}=250 \mathrm{nM}\right)$ é um conhecido inibidor de proteínas cinase que induz a apoptose, e foi utilizado como controlo positivo de citotoxicidade (26). Os resultados obtidos são apresentados na Tabela 2.

De acordo com a Tabela 2, a estaurosporina utilizada como controlo positivo resultou numa citotoxicidade dos macrófagos de $21,86 \%$ ( $p<0,0001)$. Por outro lado, os compostos testados não revelaram toxicidade significativa para os macrófagos $(p>0,05)$, uma vez que a viabilidade celular foi semelhante aos valores obtidos para células não tratadas, usadas como controlo negativo.

Os compostos 1-3 não mostraram atividade contra $M$. smegmatis (Tabela 2) (valores de CMI de $\geq 100 \mu \mathrm{g} / \mathrm{mL}$ ), quando comparados com a Rifampicina (controlo positivo, CMI 3,75 ng/mL). No entanto, em comparação com os diterpenos de labdano (2) e (3), o diterpeno halimano (1) foi o mais ativo com um valor de MIC de $100 \mu \mathrm{g} / \mathrm{mL}$, sugerindo que mais estudos adicionais são necessários, tais como a formulação farmacêutica de novos derivados do halimano puro e testes contra $M$. tuberculosis, que deveriam ser realizados para entender a atividade anti-tubercular e melhorar sua eficácia. 
Table 2/ Tabela 2 - MIC values of the compounds 1 - 3 isolated from P. ornatus, obtained by the microdilution method against Mycobacterium smegmatis. Rifampicin was used as control. The MIC values were taken as the lowest concentration of the compound that inhibited $\geq 99 \%$ the growth of the microorganisms, after $24 \mathrm{~h}$ of incubation at $37^{\circ} \mathrm{C}$, and are presented in $\mu \mathrm{g} \cdot \mathrm{mL}^{-1}$./ Valores de CMI dos compostos $1-$ 3 isolados de P. ornatus, obtidos pelo método de microdiluição contra Mycobacterium smegmatis. A Rifampicina foi utilizada como controlo positivo. Os valores de CMI

foram tomados como a menor concentração do composto que inibiu $\geq 99 \%$ do crescimento do microrganismo, após $24 \mathrm{~h}$ de incubação a $37^{\circ} \mathrm{C}$, e estão apresentados em $\mu \mathrm{g} \cdot \mathrm{mL}^{-1}$.

\begin{tabular}{cc}
\cline { 2 - 2 } & M. smegmatis \\
\hline Compound/Composto & $\mathrm{MIC}_{99} / \mathrm{CMI}_{99}\left(\mu \mathrm{g} \cdot \mathrm{mL}^{-1}\right)$ \\
\hline $\mathbf{1}$ & 100 \\
$\mathbf{2}$ & $>100$ \\
$\mathbf{3}$ & $>100$ \\
Positive & $3.75 \mathrm{RIF}$ \\
$\begin{array}{c}\text { Control/Controlo } \\
\text { positivo }\end{array}$ \\
\hline \multicolumn{2}{c}{ RIF - Rifampicin/Rifampicina }
\end{tabular}

\section{Conclusion}

Halimane [Compound 1] and labdane [Compounds 2-3] diterpenes were assessed in a preliminary assay for their anti-mycobacterial activity against $M$. smegmatis. The compounds were first tested for cytotoxicity on PBMC-derived macrophages and no cytotoxicity was found up to $25 \mu \mathrm{g} / \mathrm{mL}$. The $\mathrm{MIC}_{99}$ of Compounds 1-3 $(\geq 100 \mu \mathrm{g} / \mathrm{mL})$, did not show significant activity against $M$. smegmatis in comparison with the positive control Rifampicin. Further studies are currently underway, however, using $M$. tuberculosis in order to confirm the MIC results and in effort toin effort to elucidate the mechanism of action and improve activity with derivatives obtained by hemi-synthesis.

\section{Financial Support}

This work was supported by funding from the Italian Ministry of Health ("Ricerca Corrente, Linea 4" and "Ricerca Finalizzata, GR-2011-02350886") and the Italian Ministry of Education, Universities and Research (PRIN 20152CB22L). In addition, part of the work was carried out under a short-term scientific mission supported by COST Action CM1407 "Challeng-

\section{Conclusão}

Os diterpenos de halimano (1) e labdano (2-3) foram avaliados num ensaio preliminar da actividade antimicobacteriana contra $M$. smegmatis. Em primeiro lugar, os compostos foram testados quanto à toxicidade em macrófagos derivados de PBMC e nenhuma citotoxicidade foi encontrada até $25 \mu \mathrm{g} / \mathrm{mL}$. Os valores de $\mathrm{CMI}_{99}$ dos compostos 1-3 ( $\left.\geq 100 \mu \mathrm{g} / \mathrm{mL}\right)$, não mostraram atividade significativa contra $M$. smegmatis, em comparação com o controlo positivo de Rifampicina. Contudo, mais estudos estão a ser realizados contra $M$. tuberculosis para confirmar os resultados dos CMI, e, eventualmente elucidar o mecanismo de ação e melhorar a atividade com compostos derivados por hemisíntese.

\section{Financiamento}

Este trabalho foi apoiado pelo financiamento do Ministério da Saúde italiano ("Ricerca Corrente, Linea 4" e "Ricerca Finalizzata, GR-2011-02350886") e do Ministério Italiano do Ministério da Educação, Universidades e Pesquisa (PRIN 20152CB22L). Além disso, parte do trabalho foi realizada sob uma missão científica de curto prazo apoiada pela COST Action CM1407 
ing organic synthesis inspired by nature: from natural products chemistry to drug discovery".

\section{Acknowlegdments}

Cytotoxicity assays were developed within the framework of the Short Term Scientific Mission CM1407 (Natural Diterpenoids as potential anti-tubercular drugs) at the National Institute of Infectious Diseases Lazzaro Spallanzani, Laboratory of Cell Biology and Electron Microscopy, Rome, Italy.

\section{Ethics statement}

Human blood was collected at the Blood Bank of San Camillo Hospital (Rome, Italy), operating under license from the Italian Ministry of Health, from healthy volunteers after written informed consent.

\section{Conflict of interest}

Conflicts of interest: none. The authors declare no competing financial interests.
"Challenging organic synthesis inspired by nature: from natural products chemistry to drug discovery ".

\section{Agradecimentos}

Os ensaios de citotoxicidade foram desenvolvidos no âmbito da Missão Científica de Curto Prazo CM1407 ("Natural diterpenoids as potential anti-tubercular drugs") no Instituto Nacional de Doenças Infecciosas Lazzaro Spallanzani, Laboratório de Biologia Celular e Microscopia Eletrónica, Roma, Itália.

\section{Declaração de ética}

O sangue humano foi recolhido no Hospital do Banco do Sangue de San Camillo (Roma, Itália), operado sob licença do Ministério da Saúde de Itália, de voluntários saudáveis após consentimento informado por escrito.

\section{Conflito de interesses}

Conflitos de interesses: nenhum. Os autores declaram não haver interesses financeiros concorrentes. 


\section{References/ Referências}

(1) Meena, L.S., Rajni, K., 2010. Survival mechanisms of pathogenic Mycobacterium tuberculosis H37Rv. FEBS J. 277, $2416-2427$.

(2) Goletti D, Petruccioli E, Romagnoli A, Piacentini M, Fimia GM. 2013. Autophagy in Mycobacterium tuberculosis infection: a passepartout to flush the intruder out? Cytokine Growth Factor Rev. 24,335-43.

(3) Petruccioli E, Scriba TJ, Petrone L, Hatherill M, Cirillo DM, Joosten SA, Ottenhoff TH, Denkinger CM, Goletti D. 2016. Correlates of tuberculosis risk: predictive biomarkers for progression to active tuberculosis. Eur Respir J. 48, 1751-1763.

(4) Goletti D, Petruccioli E, Joosten SA, Ottenhoff TH. 2016. Tuberculosis Biomarkers: From Diagnosis to Protection. Infect Dis Rep. 8, 6568.

(5) Lawn, S.D., Zumla, A., 2011. Tuberculosis. Lancet 378, 57-72.

(6) Sandhu, G.K., 2011. Tuberculosis: current situation, challenges and overview of its control programs in India. J. Glob. Infect. Dis. 3, 143-150

(7) WHO. (2017). Global Tuberculosis Report 2017. Geneva.

(8) Pereira, M., Matias, D., Pereira, F., Reis, C., Simões, M.F., Rijo, P., 2015. Antimicrobial screening of Plectranthus madagascariensis and P. neochilus extracts. Biomed. Biopharm. Res. 12, 127-138.

(9) Etienne, G., Laval, F., Villeneuve, C., Dinadayala, P., Abouwarda, A., Zerbib, D., Galamba, A., Daffé, M., 2005. The cell envelope structure and properties of Mycobacterium smegmatis mc2 155: is there a clue for the unique transformability of the strain? Microbiology. 151, 2075-2086.

(10) Altaf, M., Miller, C.H., Bellows, D.S., O'Toole, R., 2010. Evaluation of the Mycobacterium smegmatis and BCG models for the discovery of Mycobacterium tuberculosis inhibitors, Tuberculosis. 90, 333-337.

(11) Akinola, R.O., Maz, G.K., Mulder, N.J., 2013. A systems level comparison of Mycobacterium tuberculosis, Mycobacterium leprae and Mycobacterium smegmatis based on functional interaction network analysis, J. Bacteriol. Parasitol. 4, 1-11.

(12) Lukhoba, C.W., Simmonds, M.S.J., Paton, A.J., 2006. Plectranthus: A review of ethnobotanical uses. J. Ethnopharmacol. 103 , 1-24.

(13) Matu, E.N., van Staden, J., 2003. Antibacterial and anti-inflammatory activities of some plants used for medicinal purposes in Kenya. J. Ethnopharmacol. $87,35-41$.

(14) Van Vuuren, S.F., 2008. Antimicrobial activity of South African medicinal plants. J. Ethnopharmacol. 119, 462-472.

(15) Abdel-Mogib, M., Albar, H.A., Batterjee, S.M., 2002. Chemistry of the Genus Plectranthus. Molecules 7, 271-301.

(16) Rijo, P., Faustino, C., Simões, M.F., 2013. Antimicrobial natural products from Plectranthus plants in Microbial Pathogens and Strategies for Combating Them: Science, Technology and Education. A. Méndez-Vilas Ed., vol. 2, 922-931.

(17) Rijo, P., Rodríguez, B., Duarte, A., Fatima Simões, M., 2011. Antimicrobial properties of Plectranthus ornatus extracts, 11-acetoxyhalima-5,13-dien15-oic acid metabolite and its derivatives. Nat. Prod. J. 1, 57-64.

(18) Rijo, P., Gaspar-Marques, C., Simões, M.F., Jimeno, M.L., Rodríguez, B., 2007. Further diterpenoid from Plectranthus ornatus and P. gradidentatus. Biochem. Syst. Ecol. 35, 215-221.

(19) Rijo, P., Gaspar-Marques, C., Simões, M.F., Duarte, A., Apreda-Rojas, M.C., Cano, F.H., Rodríguez, B., 2002. Neoclerodane and labdane diterpenoid from Plectranthus ornatus. J. Nat. Prod. 65, 1387-1390.

(20) Rijo, P., Simões, M.F., Rodríguez, B., 2005. Structural and spectral assignment of three forskolin-like diterpenoids isolated from Plectranthus ornatus. Magn. Reson. Chem. 43, 595-598.

(21) Rijo, P., Fernandes, A.S., Simões, F., Pinheiro, L., 2012. Evaluation of diterpenoids from P. ornatus as potential COX-1 inhibitors. Biomed. Biopharm. Res. 9, 111-118.

(22) Petruccioli E, Romagnoli A, Corazzari M, Coccia EM, Butera O, Delogu G,Piacentini M, Girardi E, Fimia GM, Goletti D. 2012. Specific T cells restore theautophagic flux inhibited by Mycobacterium tuberculosis in human primary macrophages. J Infect Dis. 205, 1425-35.

(23) Romagnoli, A., Etna, M.P., Giacomini, E., Pardini, M., Remoli, M.E., Corazzari, M., Falasca, L., Goletti, D., Gafa, V., Simeone, R., et al., 2012. ESX-1 dependent impairment of autophagic flux by Mycobacterium tuberculosis in human dendritic cells. Autophagy 8, 1357-1370.

(24) Rijo, P., Simões, M.F., Francisco, A.P., Rojas, R., Gilman, R.H., Vaisberg, A.J., Rodríguez, B., Moiteiro, C., 2010. Anti-mycobacterial metabolites from Plectranthus: royleanone derivatives against Mycobacterium tuberculosis strains. Chem. Biodivers. 7, 922-932.

(25) Neto, Í., Andrade, J., Fernandes, A.S., Pinto Reis, C., Salunke, J.K., Priimagi, A., Candeias, N.R., Rijo, P, 2016. Multicomponent petasis-borono mannich preparation of alkylaminophenols and antimicrobial activity studies. ChemMedChem. 11, 2015-2023.

(26) Danelishvili, L., McGarvey, J., Li, Y.J., Bermudez, L.E., 2003. Mycobacterium tuberculosis infection causes different levels of apoptosis and necrosis in human macrophages and alveolar epithelial cells. Cell. Microbiol. 5, 649-660. 